\title{
Análisis de las sentencias judiciales contra neurocirujanos resueltas en segunda instancia en España entre 1995 y 2007
}

\author{
A. Santiago-Sáez***; B. Perea-Pérez**; E. Labajo-González**; M.E. Albarrán-Juan** y J. A. Barcia*** \\ *Servicios de Medicina Legal y ***Neurocirugía. Hospital Clínico San Carlos. **Departamento de Toxicología y Legislación Sanitaria. \\ Facultad de Medicina. Universidad Complutense. Madrid.
}

Resumen

Objetivos. Con el fin de identificar los factores relacionados con las condenas judiciales contra neurocirujanos, el presente estudio analiza todas las sentencias emitidas en segunda instancia en España contra neurocirujanos en el periodo de 1995 al 2007.

Material y método. De un total de 1899 sentencias de segunda instancia o última durante el periodo de 1995 a 2007 emitidas en España se han escogido 61 que cumplen los criterios de inclusión para nuestro estudio. Se han cumplimentado 25 variables en la ficha de recogida de datos. Se ha realizado con ellas un completo estudio descriptivo y comparativo, además de un análisis de la tipología de las demandas, las circunstancias y los profesionales implicados.

Resultados. En un tercio de los casos se apreció completa mal praxis. En relación a las condenas, se absolvió aproximadamente en la mitad de los casos, siendo las resueltas con condena $1 / 5$ de ellas de carácter penal y 4/5 indemnizatorias. Las cuantías indemnizatorias predominan en el rango de 60.000 a 600.000 euros. En un 17\% de las demandas se apreció deficiencia en la información o el consentimiento. El 62,5\% de las intervenciones de nuestra muestra se produjeron en la región anatómica de la columna vertebral siguiéndole en frecuencia la región craneal con un $28,6 \%$. De las consecuencias del procedimiento quirúrgico las secuelas permanentes mayores constituyen el $40 \%$ de los casos y el fallecimiento el $22 \%$.

Conclusiones. Es rentable dedicar tiempo a la relación con el paciente, incluyendo el consentimiento informado verbal, que debe ser refrendado por la firma del documento que lo recoge. También es importante dejar constancia escrita de la praxis, tanto en lo referente a los procesos quirúrgicos como al diagnóstico y seguimiento del paciente. No se debe bajar la guardia ante procedimientos de menor riesgo vital, como la patología degenerativa, ya que son los más demandados. La

Recibido: 29-04-09. Aceptado: 3-07-09 demanda más importante tiene que ver con la satisfacción económica, especialmente en la práctica privada.

PALABRAS CLAVE: Sentencias judiciales. Condenas. Indemnizaciones. Mal praxis. Neurocirugía.

Analysis of Judicial Sentences Against Neurosurgeons Resolved in Second Court of Justice in Spain in the Period from 1995 to 2007

Summary

Objectives. With the aim of identifying the factors related to sentences against neurosurgeons, we have analyzed all the sentences issued in the second court of justice in Spain against neurosurgeons in the period from 1995 to 2007.

Material and methods. Of a total of 1899 sentences of the second or last appeal, during the period from 1995 to 2007 issued in Spain, 61 were chosen which fulfill the criteria to be included in our study. 25 variables were included on the record of compiled data. A complete descriptive and comparative study was elaborated, as well as an analysis of the type of suits, circumstances, and professionals involved.

Results. In a third of the cases, complete malpraxis was identified. In regards to the sentences, they were absolved in approximately half the cases, resolved with one fifth of the cases being penal, and four fifths with compensation. Indemnity quantities range from 60,000 to $600,000 €$. Deficiency of information or consent was noted in $17 \%$ of lawsuits. $62.5 \%$ of operations in our sample were on the anatomic region of the vertebral column followed in frequency by the cranial region with $\mathbf{2 8 . 6 \%}$. Consequences of surgical procedure included major permanent sequelae in $40 \%$ of the cases and death in $22 \%$.

Conclusions. It is wise to invest time to deal with patients, including the verbal informed consent, which must be confirmed by the written informed consent form.

It is also important to leave a written proof of medi- 
cal praxis, both related to surgical records and to diagnosis and follow-up of the patient.

Procedures with a lower life-threatening risk should not be underestimated, since they comprise the greatest demanded group.

The greatest amount of demands is related to economic reimbursement, especially in private practice.

KEY WORDS: Judicial sentences. Penalties. Indemnities. Malpraxis. Neurosurgery

\section{Introducción}

El incremento de la judicialización de los asuntos sanitarios en España afecta a todas las especialidades, sean quirúrgicas o médicas, hospitalarias o extrahospitalarias. Durante el periodo de 1995 a 1998 se presentaron contra el sistema nacional de salud 932 reclamaciones. Los errores médicos costaron al extinto Insalud 726,5 millones de las antiguas pesetas por indemnizaciones a 95 pacientes (una media de 7,8 millones de pesetas por paciente). Por especialidades, Medicina de Urgencia supuso el 23,8\% de las reclamaciones, Ginecología y Obstetricia el 18,2\%, Traumatología el 11,2\% y Neurocirugía el 4,6\% (3).

Una consecuencia inmediata de esta situación puede ser la práctica sistemática de la medicina defensiva, que se aleja de la relación fluida entre médico y paciente, basada en la confianza mutua y con una orientación bioética que potencia la propia autonomía del paciente. Sin embargo, un análisis de los factores que motivan una demanda y de los condicionamientos que influyen en las decisiones judiciales puede ayudar a los especialistas en neurocirugía a valorar determinadas prácticas clínicas de forma especial, extremando las precauciones ante aquellos signos que puedan dar lugar a una demanda judicial.

En este artículo pretendemos determinar las características de las demandas presentadas contra médicos especialistas en neurocirugía, considerando todas aquellas variables que pueden tener alguna influencia tanto en el planteamiento de la demanda como en la resolución del proceso judicial. El objetivo es analizar las demandas judiciales presentadas contra médicos especialistas en neurocirugía, estableciendo la tipología del procedimiento quirúrgico demandado y analizando la resolución de las sentencias, considerando si existe condena y su cuantía penal o económica. De este modo, los resultados podrán facilitar una práctica más segura de la especialidad, tanto para el paciente como para el médico. No existe en la literatura de los últimos años ningún estudio similar, que haga referencia específica a la especialidad de neurocirugía en España.

\section{Material y método}

El presente estudio se ha realizado sobre el "Archivo de sentencias judiciales sanitarias de la Escuela de Medicina Legal de Madrid", de la Universidad Complutense de Madrid. Este archivo contiene 1899 sentencias judiciales de segunda o última instancia referidas a actuaciones sanitarias emitidas por las jurisdicciones Civil, Penal y Contencioso-administrativa durante el periodo comprendido entre 1995 y junio de 2007. El archivo contiene 61 sentencias que han sido consideradas para este estudio en base a los siguientes criterios de inclusión:

1. Sentencia referida a un médico especialista en neurocirugía, debido al ejercicio de su especialidad, o sentencia referida a un médico en la que no consta su especialidad, pero en la que la maniobra reclamada judicialmente corresponde a la especialidad de neurocirugía, según definición y maniobras contempladas en el anexo a la orden SCO/847/2008, de 14 de marzo, por la que se aprueba y regula el programa formativo de la especialidad de neurocirugía.

2. Texto de la sentencia en el que figure el tratamiento reclamado, las circunstancias y lugar en el que se produjo, sus consecuencias y la resolución judicial.

En la ficha de recogida de datos se han implementado las siguientes variables:

1. Clave de identificación en la base de Aranzadi (referencia)

2. Clave de identificación en el presente proyecto de investigación

3. Jurisdicción en la que se dirime la demanda

Civil

Penal

Contencioso-administrativa

Otras

4. Tipo de tribunal que dicta la sentencia

Audiencia provincial

Tribunal Superior de Justicia

Tribunal Supremo

Otros

5. Provincia donde se produce la demanda

6. Fecha de la sentencia

7. Fallo de la sentencia

Apreciación completa de mal praxis

Apreciación parcial de mal praxis

No apreciación de mal praxis

Otras

8. Existencia de deficiencias de información o consentimiento

9. Referencia a la obligación de medios o resultados

10. Tipo de condena

Penal

Indemnización económica

11. Cuantía de la indemnización 
12. Género del paciente

13. Si el paciente se trata de un menor

14. Circunstancias añadidas al proceso patológico

15. Actividad específica por región anatómica

Cráneo

Columna

Miembros

16. Actividad específica por tipo de lesión intervenida

Vascular

Tumoral

Degenerativa

Otras

17. Fecha de atención sanitaria reclamada

18. Número y clase de profesionales reclamados

Un único profesional

Más de un profesional de una especialidad

Profesionales de varias especialidades

19. Existencia de entidades de asistencia sanitaria demandadas

20. Género del profesional

21. Lugar de asistencia sanitaria

Hospital público

Centro de salud público

Hospital privado

Centro sanitario privado sin internamiento

22. Hecho imputado

Error diagnóstico

Error terapéutico (farmacológico o quirúrgico)

Error de seguimiento (alta o revisiones)

Error o deficiencias en la información

Accidente

Complicación

23. Daños y secuelas

24. Etiología de la lesión

Traumática

Tumoral

Degenerativa

Otras

25. Existencia o no de explicación escrita

Se ha realizado un análisis de contingencia entre las distintas variables mediante la prueba Chi cuadrado utilizando el programa estadístico SPSS 15. Por otra parte, se ha realizado un análisis de agregación multifactorial con la aplicación SPADN ("Système Portable pour l'Analyse des Données". Ver. 5.6, CISIA, Montreuil Cedex. France, 2003), para identificar asociaciones entre las variables. Se ha establecido un nivel de significancia estadística del $95 \%$ $(\mathrm{p}<0,05)$.

\section{Resultados}

En 40 casos ( $80 \%$ de los 50 que recogen este dato) el cirujano imputado era varón, en 1 caso mujer (2\%), y en

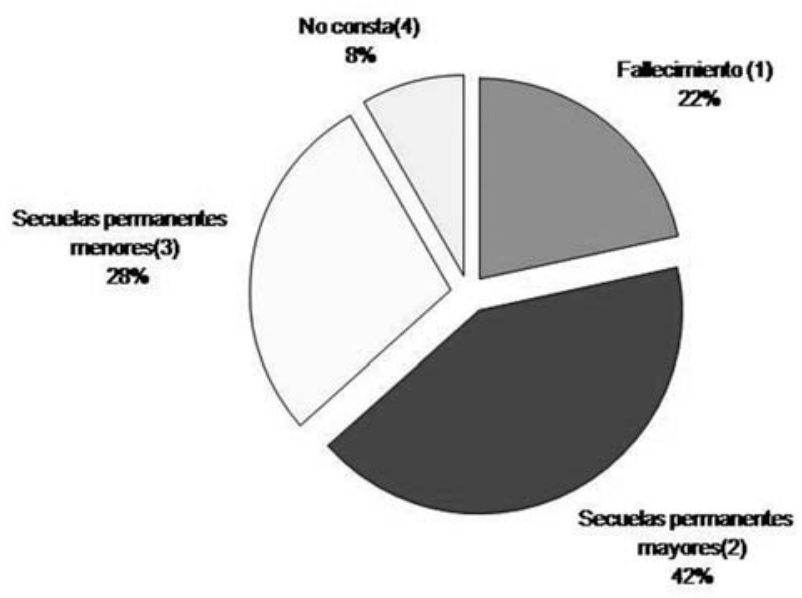

Figura 1. Porcentaje de las sentencias judiciales contra neurocirujanos resueltas en segunda instancia en España entre 1995 y 2007, en función del resultado del procedimiento quirúrgico o la asistencia.

9 casos hubo imputados de ambos géneros (18\%). En 31 casos se trató de un especialista en neurocirugía (31\% de los 48 que recogen este dato), en 9 (18,8\%) más de un neurocirujano y en $8(16,6 \%)$ se trató de especialistas de varias especialidades. En 52 (casos 92,9\% de 56) se demandó además a la entidad que prestaba asistencia sanitaria.

En la tabla 1 se recogen el número de sentencias en función de la jurisdicción que dirime el caso, el fallo de la sentencia, la cuantía de la indemnización y el lugar de asistencia. En prácticamente la mitad de los casos de la muestra se produce una condena (29 de las 61 sentencias) siendo el $82,8 \%$ de las condenas de carácter indemnizatorio, y de éstas la cuantía más frecuente en un 59,3\% de los casos es de 60.000 a 600.000 euros, siendo el ámbito de asistencia el hospital público en un 70,2\% de los casos. Existe una relación estadísticamente significativa entre el tipo de jurisdicción y la cuantía de la indemnización, destacando indemnizaciones entre $60.000 €$ y $600.000 €$ y más de $600.000 €$ en la vía jurisdiccional civil. También existe una relación estadísticamente significativa entre el tipo de tribunal y la cuantía de la indemnización, destacando indemnizaciones entre 60.000€ y 600.000€ en la Audiencia Provincial y Tribunal Supremo frente al TSJ, en porcentajes muy similares. Las dos sentencias mayores de 600.000€ corresponden una a la Audiencia Provincial y otra al Tribunal Supremo. Por otra parte, se encuentra una relación estadísticamente significativa entre el lugar de la asistencia sanitaria y la cuantía de la indemnización, destacando las indemnizaciones entre 60.000€ y 600.000€ en los Hospitales públicos. Una de las sentencias mayores de $600.000 €$ corresponde a un centro privado sin internamiento. 
Tabla 1

Número de sentencias en función de la jurisdicción que dirime el caso, el fallo de la sentencia, la cuantía de la indemnización y el lugar de asistencia

\begin{tabular}{|c|c|c|c|}
\hline & & Número & Porcentaje \\
\hline \multirow{3}{*}{$\begin{array}{l}\text { Jurisdicción } \\
\text { Total: } 60\end{array}$} & Civil & 48 & 80,0 \\
\hline & Penal & 7 & 11,7 \\
\hline & Contencioso-Administrativa & 5 & 8,3 \\
\hline \multirow{3}{*}{$\begin{array}{l}\text { Tribunal } \\
\text { Total: } 60\end{array}$} & Audiencia Provincial & 29 & 48,3 \\
\hline & Tribunal Superior de Justicia & 5 & 8,3 \\
\hline & Tribunal Supremo & 26 & 43,3 \\
\hline \multirow{13}{*}{$\begin{array}{l}\text { Comunidad Autónoma } \\
\text { Total: } 32\end{array}$} & Andalucía & 6 & 18,8 \\
\hline & Aragón & 1 & 3,1 \\
\hline & Asturias & 1 & 3,1 \\
\hline & Baleares & 2 & 6,3 \\
\hline & C. Valenciana & 4 & 12,5 \\
\hline & Canarias & 1 & 3,1 \\
\hline & Castilla León & 1 & 3,1 \\
\hline & Cataluña & 4 & 12,5 \\
\hline & Extremadura & 2 & 6,3 \\
\hline & Galicia & 4 & 12,5 \\
\hline & Madrid & 4 & 12,5 \\
\hline & Murcia & 1 & 3,1 \\
\hline & P. Vasco & 1 & 3,1 \\
\hline \multirow{3}{*}{$\begin{array}{l}\text { Fallo de sentencia } \\
\text { Total: } 60\end{array}$} & Completa mal praxis & 17 & 28,3 \\
\hline & Parcial mal praxis & 9 & 15,0 \\
\hline & No mal praxis & 34 & 56,7 \\
\hline \multirow{7}{*}{$\begin{array}{l}\text { Condena } \\
\text { Total: } 29 \\
\text { Cuantía de la indemnización } \\
\text { Total: } 27\end{array}$} & Penal & 5 & 17,2 \\
\hline & Indemnización & 24 & 82,8 \\
\hline & Menos de $6.000 €$ & 0 & 0 \\
\hline & $6.000-18.000 €$ & 3 & 11,1 \\
\hline & $18.000-60.000 €$ & 6 & 22,2 \\
\hline & $60.000-600.000 €$ & 16 & 59,3 \\
\hline & Mas $600.000 €$ & 2 & 7,4 \\
\hline \multirow{4}{*}{$\begin{array}{l}\text { Lugar de asistencia } \\
\text { Total: } 57\end{array}$} & Hospital público & 40 & 70,2 \\
\hline & Centro de Salud público & 0 & 0 \\
\hline & Hospital privado & 13 & 22,8 \\
\hline & Centro sin internamiento privado & 4 & 7 \\
\hline
\end{tabular}

No existe en nuestra muestra diferencias en cuanto al género del paciente, tratándose en un $8,5 \%$ de los casos de menores de edad.

En la tabla 2 se recoge el tipo de sentencia respecto a las variables referentes al proceso asistencial y a las consecuencias del procedimiento quirúrgico o la asistencia. Llama la atención la existencia de una relación significativa entre la referencia a deficiencias en la información y consentimiento y el fallo de la sentencia, destacando la no apreciación de mal praxis en los casos en que no existen deficiencias en la información y consentimiento.

El análisis de agregación multifactorial mostró cuatro asociaciones entre factores con significado clínico que pueden constituir tipos característicos de condenas:

1. Sentencias en las que no se aprecia mal praxis, con intervenciones en la región anatómica de la columna, imputándose errores terapéuticos, en pacientes asistidos en hospital privado, juzgados en la audiencia provincial, sin referencia a deficiencias en la información o el consentimiento, en patologías de etiología degenerativa. $31,67 \%$ de las sentencias se encuentran en este grupo con un $95 \%$ de probabilidad.

2. Lesión intervenida de tipo vascular, en la región craneal o en miembros, con etiología del proceso traumática 
Tabla 2

Estudio de frecuencias respecto a las variables referentes al proceso asistencial y las consecuencias del procedimiento quirúrgico o la asistencia.

\begin{tabular}{|c|c|c|c|}
\hline & & Número & Porcentaje \\
\hline \multirow{2}{*}{$\begin{array}{l}\text { Deficiencias información o consentimiento } \\
\text { Total: } 60\end{array}$} & $\mathrm{Si}$ & 10 & 16,7 \\
\hline & No & 50 & 83,3 \\
\hline \multirow{3}{*}{$\begin{array}{l}\text { Obligación de. ¿? } \\
\text { Total: } 60\end{array}$} & Resultados & 0 & 0 \\
\hline & Medios & 23 & 38,3 \\
\hline & No consta & 37 & 61,7 \\
\hline \multirow{2}{*}{$\begin{array}{l}\text { Sexo del paciente } \\
\text { Total: } 58\end{array}$} & Hombre & 30 & 51,7 \\
\hline & Mujer & 28 & 48,3 \\
\hline \multirow{2}{*}{$\begin{array}{l}\text { Menor de edad } \\
\text { Total: } 59\end{array}$} & $\mathrm{Si}$ & 5 & 8,5 \\
\hline & No & 54 & 91,5 \\
\hline \multirow{3}{*}{$\begin{array}{l}\text { Región anatómica } \\
\text { Total: } 56\end{array}$} & Cráneo & 16 & 28,6 \\
\hline & Columna & 35 & 62,5 \\
\hline & Miembros & 5 & 8,9 \\
\hline \multirow{4}{*}{$\begin{array}{l}\text { Lesión Intervenida } \\
\text { Total: } 54\end{array}$} & Vascular & 12 & 22,2 \\
\hline & Tumoral & 7 & 13 \\
\hline & Degenerativa & 30 & 55,5 \\
\hline & Otras & 5 & 9,3 \\
\hline \multirow{4}{*}{$\begin{array}{l}\text { Hecho imputado } \\
\text { Total: } 58\end{array}$} & Error diagnóstico & 12 & 20,7 \\
\hline & Error terapéutico & 32 & 55,2 \\
\hline & Error de seguimiento & 8 & 13,8 \\
\hline & Déficit información & 6 & 10,3 \\
\hline \multirow{4}{*}{$\begin{array}{l}\text { Etiología } \\
\text { Total: } 57\end{array}$} & Traumática & 9 & 15,8 \\
\hline & Tumoral & 11 & 19,3 \\
\hline & Degenerativa & 30 & 52,6 \\
\hline & Otras & 7 & 12,3 \\
\hline \multirow{3}{*}{$\begin{array}{l}\text { Explicación escrita } \\
\text { Total: } 59\end{array}$} & $\mathrm{Si}$ & 7 & 11,9 \\
\hline & No & 10 & 16,9 \\
\hline & No consta & 42 & 71,2 \\
\hline
\end{tabular}

o considerada como "otras causas", con condena de indemnizaciones económicas de 60.000 a 600.000 euros, en la jurisdicción civil y haciéndose referencia a la obligación de medios: $25 \%$ de las sentencias.

3. Etiología degenerativa, con actividad específica en patología degenerativa, con apreciación completa de mal praxis, en región anatómica de columna, en condenas del tribunal supremo, contra un solo profesional, con resultado de condenas indemnizatorias y penales en cuantías de 18.000 a 60.000 euros por error terapéutico o de seguimiento y en la jurisdicción civil (tabla 1): $25 \%$ de los casos.

4. Jurisdicción contencioso-administrativa, en sede de Tribunales Superiores de Justicia, con existencia de explicación escrita del procedimiento, con indemnizaciones de 6.000 a 18.000 euros y deficiencias en la información y el consentimiento: $8,33 \%$ de los casos.

5. Demandas sin presencia de entidades de asistencia sanita- ria, en la jurisdicción penal, por imputación de error diagnóstico, por actividad quirúrgica en la región anatómica craneal y de etiología traumática: $5 \%$ de los casos.

\section{Discusión}

La neurocirugía es una especialidad de alto riesgo judicial, situándose en el cuarto lugar del total de demandas por especialidad en España. En otros países también ocupa un lugar destacado ${ }^{7}$. El número de reclamaciones anuales contra los servicios de neurocirugía de tamaño medio que publican sus estadísticas oscila entre 40 y 84 . El estudio del periodo 1995-2000 del INSALUD ${ }^{3}$ arroja un coste medio de indemnizaciones por caso en la especialidad de neurocirugía de $126.000 €$.

El análisis de las sentencias muestra:

1. La mayoría de las demandas que prosperan tienen como objetivo la compensación económica. Respecto a 
la demanda el $80 \%$ de la muestra total es de orden civil, encontrándose solo en el orden penal el $11 \%$ de los casos. Este estudio recoge las sentencias en segunda instancia o última instancia contra neurocirujanos, lo que quiere decir que en el orden penal muchas demandas o no se admiten a trámite y se archivan o no se condenan en primera instancia, puesto que el volumen de demandas presentadas en la ámbito penal es muy superior. Según el análisis de agregación, el 31,67\% de los casos de la muestra entran con un $95 \%$ de probabilidad en el tipo de sentencias en las que no se aprecia mal praxis, sin referencia a deficiencias en la información o el consentimiento, imputándose errores terapéuticos, en pacientes asistidos en hospitales privados con cirugía por patología degenerativa de raquis, juzgados en la audiencia provincial. De las demandas que llegan a segunda instancia el $48,3 \%$ se dirimen en la Audiencia Provincial y el $43,3 \%$ en el Tribunal Supremo. Destaca como Comunidad Autónoma la Andaluza con un 18,8\% de los casos, aunque las demandas se encuentran muy repartidas en nuestra geografía existiendo casos en 13 de las 18 Comunidades Autónomas. Las sentencias de los tribunales de segunda instancia como la Audiencia Provincial o el Tribunal Supremo en sus salas de lo civil se relacionan de forma significativa con las indemnizaciones más elevadas, en el rango de 60.000 a $600.000 €$, y en el ámbito de la asistencia hospitalaria pública. Posiblemente el límite de los $600.000 €$ tiene relación con el límite que establecen las compañías en las indemnizaciones por siniestro. En las demandas se imputa en el 64,6\% a un solo profesional, y en el 92,9\% están implicadas entidades de asistencia sanitaria (ya sean mutuas, compañías aseguradoras o entidades privadas de asistencia).

2. La patología degenerativa del raquis es el blanco más importante de las demandas. El $62,5 \%$ de las intervenciones de nuestra muestra se produjeron en la región anatómica de la columna vertebral siguiéndole en frecuencia la región craneal con un $28,6 \%$. Respecto al tipo de lesión intervenida, ésta fue en su mayoría patología degenerativa del raquis (55,5\% de los casos). El $25 \%$ de la muestra entra la siguiente combinación: etiología degenerativa, con actividad específica en región anatómica de columna, con apreciación completa de mal praxis, en condenas del tribunal supremo, contra un solo profesional, con resultado de condenas indemnizatorias y penales en cuantías de 18.000 a 60.000 euros por error terapéutico o de seguimiento y en la jurisdicción civil. En el estudio realizado en el Servicio Nacional de Salud del Reino Unido en el periodo de 1995 a 2005 en Newcastle ${ }^{5}$ se estudiaron 559 casos de los cuales neurocirugía se denunció por negligencia en 241 casos, por procesos patológicos el porcentaje fue el siguiente: enfermedad del disco intervertebral $27 \%$, tumores del SNC $21 \%$, infección del SNC 11\% y hemorragia subaracnoidea $9 \%$. Datos intermedios se publican en un estudio realizado en Burlington (USA) en el año 2006 sobre una muestra de 275 casos de reclamaciones por malpraxis, el $42 \%$ correspondían a cirugía espinal, datos similares a un estudio realizado en 1985 sobre una muestra de 300 reclamaciones por malpraxis ${ }^{2}$. En el estudio sobre un periodo de cinco años sobre 280 casos desde el 1 de enero de 1999 al 30 de diciembre de 2003 del New York Medical College, se obtuvieron los siguientes resultados tras examinar la demanda, el lugar anatómico y la resolución del caso: 156 (56\%) correspondían a la columna, $109(39 \%)$ la cabeza y/o el cerebro, quedando 15 casos $(5 \%)$ en una miscelánea ${ }^{6}$.

3. La documentación del consentimiento informado no protege contra las demandas, pero su ausencia las agrava. En un $83,3 \%$ de las demandas no se aprecia deficiencia en la información y el consentimiento, pero en el $16,7 \%$ restante se hace referencia a estas deficiencias. Esta documentación protege contra la acusación de mal praxis. Existe una relación significativa entre la referencia a deficiencias en la información y consentimiento y el fallo de la sentencia, destacando la no apreciación de mal praxis en los casos en que no existen deficiencias en la información y consentimiento, lo que viene a afianzar la corriente de la relación entre una práctica segura y la buena comunicación con el paciente. En este sentido, el documento legal que hace que el paciente refleje por escrito que ha sido informado y que consiente con el procedimiento no evita las demandas. De hecho, no es la firma del documento lo que constituye el consentimiento informado, sino el asentimiento del paciente a una información detallada. La firma del documento es sólo la constancia de esta. Un documento firmado en el que se demuestre que no existió comprensión por parte del paciente, o bien que se hizo con premura, puede ser invalidado. Por tanto, el consentimiento informado no tiene validez, o al menos, eficacia, si no existe una relación fluida entre el paciente y el médico. Las deficiencias en la información y el consentimiento se asocian a acusaciones en la Jurisdicción contencioso-administrativa, en sede de Tribunales Superiores de Justicia, con existencia de explicación escrita del procedimiento, con indemnizaciones de 6.000 a $18.000 €$, con el $8,33 \%$ de la muestra. No se tiene constancia de la existencia o no de información escrita en el $71,2 \%$ de los casos demandados ya que la sentencia no hace mención a ella por no ser probablemente sujeto de daño jurídico.

4. Las secuelas permanentes son más demandadas que el fallecimiento. En el análisis de nuestra muestra sobre las consecuencias del procedimiento, el fallecimiento se produjo en $22 \%$ de los casos y las secuelas permanentes mayores en el $42 \%$. En el estudio realizado en Newcastle ${ }^{5}$ las consecuencias fueron lesiones permanentes en el $47 \%$ de los casos y la muerte en el 17\%, datos similares a nuestra muestra, con un coste total de las indemnizaciones de 37 millones de libras. En el estudio del New York Medical 
College $^{6}, 98$ de los casos supusieron una indemnización total de 50 millones de dólares, mientras que 182 casos no se indemnizaron.

5. El error diagnóstico o de seguimiento puede ser tan importante como el error terapéutico. De los hechos imputados, los errores terapéuticos aparecen en el 55,2\% de los casos, el error diagnóstico supone un $20,7 \%$ de los casos, el error de seguimiento un 13,8\% y el déficit de información un $10,3 \%$. En el estudio realizado en Newcastle ${ }^{5}$ la causa más frecuente de denuncia fue por error diagnóstico (44\%). El $56,7 \%$ de los casos fueron sentenciados sin apreciar mal praxis, pero en un $28,3 \%$ si se apreció por el tribunal una completa mal praxis. El 5\% de la muestra presenta la siguiente combinación: demandas sin presencia de entidades de asistencia sanitaria, en la jurisdicción penal, por imputación de error diagnóstico, por actividad quirúrgica en la región anatómica craneal y de etiología traumática.

6. La obligación de medios puede ser más relevante que la obligación de resultados. En un 38,3\% de las sentencias se hace referencia a la obligación de medios, no existiendo sentencia alguna que haga referencia a la obligación de resultados, ya que los procesos intervenidos no se encuadran en la llamada cirugía satisfactiva o de resultados. El $25 \%$ de la muestra entra en la combinación de lesión intervenida de tipo vascular, en la región craneal o en miembros, con etiología del proceso traumática o considerada como "otras causas", con condena de indemnizaciones económicas de 60.000 a 600.000 euros, en la jurisdicción civil y haciéndose referencia a la obligación de medios.

El alto riesgo judicial de la neurocirugía podría ocasionar una reacción por parte del especialista planteando una medicina defensiva, aumentando el número de pruebas diagnósticas, enfatizando el riesgo vital de las intervenciones y, posiblemente, distanciándose del paciente. Sin embargo, la actitud defensiva no parece ser la solución a este problema. Un análisis de las demandas hecha en EE.UU. ${ }^{4}$ demostró que un factor independiente que distinguía la existencia o no de demandas era el número de minutos dedicado por el médico a la entrevista, siendo la media de 15.0 en los demandados y de 18.3 en los no demandados. Factores involucrados en la forma de la relación entre el médico y el enfermo, como el tono de voz, también parecen ser determinantes ${ }^{1}$.

Podríamos concluir con una serie de recomendaciones derivadas de este estudio:

1. Es rentable dedicar tiempo a la relación con el paciente, incluyendo el consentimiento informado verbal, que debe ser refrendado por la firma del documento que lo recoge.
2. Es importante dejar constancia escrita de la praxis, tanto en lo referente a los procesos quirúrgicos como al diagnóstico y seguimiento del paciente.

3. No se debe bajar la guardia ante procedimientos de menor riesgo vital, como la patología degenerativa, ya que son los más demandados.

4. La demanda más importante tiene que ver con la satisfacción económica, especialmente en la práctica privada. Por eso es importante disponer de un seguro de responsabilidad civil con una prima adecuada, con carácter retroactivo, y que disponga de una cobertura prolongada en el tiempo.

\section{Bibliografía}

1. Ambady, N., Laplante, D., Nguyen, T., et al.; Surgeons' tone of voice: a clue to malpractice history. Surgery. 2002; 132: 5-9.

2. Fager, C.A.: Malpractice issues in neurological surgery. Surg Neurol. 2006; 65: 416-421.

3. INSALUD, Memoria Estadistica INSALUD. Madrid; Ministerio de Sanidad y Consumo, 1999.

4. Levinson, W., Roter, D.L., Mullooly, J.P., Dull, V.T., Frankel, R.M.: Physician-patient communication. The relationship with malpractice claims among primary care physicians and surgeons. JAMA. 1997; 277: 553-559.

5. McNeill, A.: Neurological negligence claims in the NHS from 1995 to 2005. Eur J Neurol. 2007; 14: 399-402.

6. Rovit, R.L., Simon, A.S., Drew, J., Murali, R., Robb, J.: Neurosurgical experience with malpractice litigation: an analysis of closed claims against neurosurgeons in New York State, 1999 through 2003. J Neurosurg. 2007; 106: 1108-1114.

7. Studdert, D.M., Mello, M.M., Gawande, A.A., et al:. Claims, errors, and compensation payments in medical malpractice litigation. N Engl J Med 2006; 354: 2024-2033

Santiago-Sáez, A.; Perea-Pérez, B.; Labajo-González, E.; Albarrán-Juan, M.E.; Barcia, J.A.: Análisis de las sentencias judiciales contra neurocirujanos resueltas en segunda instancia en España entre 1995 y 2007. Neurocirugía 2010; 21: 53-60.

Correspondencia: Andrés Santiago Sáez. Servicio de Medicina Legal. Hospital Clínico San Carlos de Madrid

C/ Prof. Martín Lagos s/n 28040 Madrid.

E-mail: asantiago.hcsc@salud.madrid.org

Juan A. Barcia. Servicio de Neurocirugía. Hospital Clínico San Carlos de Madrid. C/ Prof. Martín Lagos s/n 28040 Madrid.

E-mail: jabarcia.hcsc@salud.madrid.org 
Comentario al trabajo "Análisis de las sentencias judiciales contra neurocirujanos resueltas en segunda instancia en España entre 1995 y 2007” de A. Santiago Saez y cols.

En el artículo titulado "Análisis de las sentencias judiciales contra neurocirujanos resueltas en segunda instancia en España entre 1995 y 2007", los autores realizan una extensa búsqueda entre las 1899 sentencias judiciales en segunda instancia disponibles en el Archivo de sentencias judiciales sanitarias de la Escuela de Medicina Legal de Madrid, centrando su análisis sobre 61 sentencias que afec$\tan$ directamente a neurocirujanos o que son consecuencia de la práctica neuroquirúrgica, cuando por ejemplo, implica también a otros especialistas o en los casos que no se especifica la especialidad del demandado.

En un tercio de los casos analizados se apreció completa malpraxis y en un 17\% de las demandas se observó deficiencia en la información o en el consentimiento informado. En este sentido es destacable la afirmación de los autores: "la
2010; 21: 53-60

documentación del consentimiento informado no protege contra las demandas pero su ausencia las agrava".

Otro hecho destacable es que el error diagnóstico o de seguimiento puede ser judicialmente tan importante como el error terapéutico, recordándonos que nuestra responsabilidad no termina donde acaba el acto quirúrgico. También es bastante remarcable que la obligación de medios puede ser más relevante que la obligación de resultados. En este sentido se contempla la posibilidad de errar en los objetivos terapéuticos planteados pero se considera inaceptable intentar conseguir esos objetivos sin los recursos suficientes que garanticen que el procedimiento terapéutico se realiza en condiciones razonables.

Los autores concluyen con un conjunto de recomendaciones que debemos tener siempre presentes si queremos minimizar los "riesgos judiciales" hoy por hoy casi inevitables en nuestra práctica clínica.

J. Domínguez Baez Tenerife 